\title{
Joint Hypermobility Syndrome Affects Response to a Low Fermentable Oligosaccharide, Disaccharide, Monosaccharide and Polyol Diet in Irritable Bowel Syndrome Patients: A Retrospective Study
}

\author{
Konstantinos C. Fragkos ${ }^{\mathrm{a}}$, Katie Keetarut ${ }^{\mathrm{b}}$, Anna Cox ${ }^{\mathrm{a}}$, Johanna Eady ${ }^{\mathrm{b}}$, \\ Anton V. Emmanuel ${ }^{\mathrm{a}}$, Natalia Zarate-Lopez ${ }^{\mathrm{a}, \mathrm{c}}$
}

\begin{abstract}
Background: The low fermentable oligosaccharide, disaccharide, monosaccharide and polyol (FODMAP) diet causes significant clinical improvement in patients with irritable bowel syndrome (IBS). Joint hypermobility syndrome (JHS), defined as musculoskeletal symptoms in a hypermobile individual in the absence of systemic rheumatological disease, may be associated with functional gastrointestinal symptoms, including IBS. The aim of this study is to examine whether JHS can affect the response to the low FODMAP diet in patients with IBS.
\end{abstract}

Methods: In this retrospective study, we included patients with IBS according to Rome III criteria who had followed a low FODMAP diet. Symptoms scores were measured before and after the low FODMAP diet.

Results: A total of 165 patients (130 females, age $44 \pm 14$ years) were included. Diarrhea predominant IBS (IBS-D) was present in $40.6 \%$ of our patients while JHS was present in $21.2 \%$. The score for abdominal pain was higher for JHS compared to non-JHS prior to intervention $(\mathrm{P}=0.011)$. Symptoms improved in both groups of patients after a low FODMAP diet $(\mathrm{P}<0.0001)$. The largest effects were shown with significant decreases of the average score and bloating. When broken down by JHS and IBS type, a low FODMAP diet significantly improved pain, bloating, diarrhea, constipation, and the average score with the largest effect in JHS/constipation predominant IBS (IBS-C), JHS/mixed IBS and unclassified IBS (IBS-M), JHS/IBS-D, non-JHS/

Manuscript submitted December 29, 2018, accepted January 16, 2019

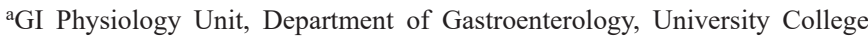
London Hospitals NHS Foundation Trust, 250 Euston Road, London NW1 2PG, UK

bDietetics Department, University College London Hospitals NHS Foundation Trust, 250 Euston Road, London NW1 2PG, UK

${ }^{\mathrm{c} C o r r e s p o n d i n g ~ A u t h o r: ~ N a t a l i a ~ Z a r a t e-L o p e z, ~ G I ~ P h y s i o l o g y ~ U n i t, ~ D e p a r t-~}$ ment of Gastroenterology, University College London Hospitals NHS Foundation Trust, 250 Euston Road, London NW1 2PG, UK.

Email: N.Zarate-Lopez@nhs.net

doi: https://doi.org/10.14740/gr1133
IBS-C and JHS/IBS-M, respectively.

Conclusions: Our study suggests that a low FODMAP diet has a greater effect on IBS symptoms in JHS than non-JHS patients.

Keywords: Joint hypermobility syndrome; Irritable bowel syndrome; FODMAP; Pain; Bloating

\section{Introduction}

Irritable bowel syndrome (IBS) is a chronic functional bowel disease with high prevalence in the general population [1]. The prevalence of IBS ranges between $7 \%$ and $21 \%$ depending on the country and criteria used, and it has an adverse effect on the patient's quality of life [2]. Four IBS subtypes are suggested by the Rome IV criteria based on stool form: diarrhea predominant IBS (IBS-D), constipation predominant IBS (IBS-C), mixed IBS and unclassified IBS (IBS-M) [3]. IBS-D is considered the most common subtype with a prevalence of $40-60 \%$ of all IBS $[4,5]$. Treatment of IBS depends on the predominant symptom that the individual is experiencing [6].

There is increased recognition that dietary factors have a significant role to play in the development of IBS symptoms [7]. Fermentable oligosaccharides, disaccharides, monosaccharides and polyols (FODMAPs) are fermented rapidly by gut microflora, slowly absorbed and osmotically active. Increased osmotic load and luminal changes secondary to gas induced distension will cause IBS symptoms in some patients $[8,9]$. Patients with IBS have heightened gut sensitivity and/or altered motility and might experience symptoms such as diarrhea, excessive flatulence, bloating and/or abdominal cramps when exposed to foods rich in FODMAPs. The associated visceral hypersensitivity explains symptom development in response to FODMAP-containing foods [10]. Recent evidence supports the implementation of the low FODMAP diet for the treatment of IBS patients and it is indeed now considered as a first-line dietetic intervention [11, 12]. Many studies have demonstrated that the low FODMAP diet achieves a significant improvement in symptoms such as bloating, abdominal 
pain and flatulence in IBS patients compare to a standard diet [13-15].

Functional gastrointestinal disorders are reported to be more common in patients with a non-inflammatory connective tissue disorder known as benign joint hypermobility syndrome (JHS) $[16,17]$. JHS is defined as musculoskeletal symptoms in a hypermobile individual in the absence of systemic rheumatological disease, and its prevalence is around $3 \%$ of the population $[18,19]$. An essential feature of JHS is generalized joint hypermobility, which refers to the characteristic of being able to actively and/or passively move joints beyond normal limits [20]. Generalized joint hypermobility can be identified in some well-defined inherited connective tissue disorders, including Ehlers-Danlos syndrome [21], Marfan syndrome and osteogenesis imperfecta [17]. Joint hypermobility can affect a few joints (localized or monoarticular joint hypermobility) or several joints in multiple body sites. Generalized joint hypermobility, in the absence of other symptoms, is considered to be a harmless trait $[22,23]$. The definitive diagnosis of JHS requires fulfilling the Brighton criteria, but generalized joint hypermobility can be screened for using a simple validated 5-point questionnaire or using the Beighton score (Supplementary Table 1) (www.gastrores.org).

The systemic complications of JHS include altered pain processing, autonomic dysfunction and chronic fatigue [24]. Patients with co-existent JHS and gastrointestinal symptoms experience mostly abdominal pain, bloating and nausea, followed by reflux symptoms, vomiting, constipation and diarrhea [25]. Fikree et al [26] reported that JHS patients referred from rheumatology clinics with gastrointestinal symptoms were significantly younger than the non-JHS patients and predominately female $(95.5 \%)$. JHS patients complain of multiple gastrointestinal symptoms although mechanisms for this remain unknown. Chronic exposure to pain and visceral hypersensitivity with altered pain processing might be involved.

Clinical management of functional gastrointestinal symptoms in patients with JHS can be challenging, particularly in patients with associated extra-intestinal chronic functional symptoms and pain. Food restriction and dysfunctional psychology can therefore lead to poor nutritional status and weight loss that can worsen the symptoms of JHS resulting in worsening fatigue and pain. Nutritional and lifestyle interventions (e.g. regular fiber intake or restrictions of foods contributing to symptoms) are a recommended therapeutic approach in JHS with functional gastrointestinal symptoms [18]. Hence, the purpose of the current study is to examine the efficacy of a low FODMAP diet in patients with IBS according to the presence of JHS.

\section{Materials and Methods}

\section{Population and settings}

This study was based at University College London Hospitals (UCLH) NHS Foundation Trust, a national referral center of congenital connective tissue disorders and a tertiary neurogastroenterology referral center. Participants were consecutively recruited from patients who visited the Gastroenterology Department at UCLH between January 2012 and June 2015. Individual patient data was anonymized, and regulatory approval was granted by the site institutional review board (2015). This study involved the retrospective review of existing data and records, and was considered a service evaluation study. Hence written, informed consent was waived and ethical approval was not required, according to the decision of institutional review board. The study protocol conforms to the ethical guidelines of the 1975 Declaration of Helsinki as reflected in a priori approval by the institutional review board. STROBE guidelines for observational studies were followed in the present study [27].

\section{Inclusion and exclusion criteria}

Included patients were 18 years old or over, had a diagnosis of IBS according to Rome III criteria confirmed by a gastroenterologist, were medically safe for a low FODMAP intervention and willing to comply with the dietary protocol. Exclusion criteria were loss to follow-up, pregnancy, patients who had undergone previous upper gastrointestinal surgery that may affect dietary intake and any other medical condition that prevented patients following a food restricted diet. Data were collected retrospectively through UCLH electronic patients' records and the IBS databases held by the neurogastroenterology team.

\section{Data collection}

The following data were collected.

\section{Demographics and IBS diagnosis}

Age, gender, and IBS subtype, based on Rome III criteria (diarrhea, constipation or mixed).

\section{Hypermobility}

Patients with a positive diagnosis made by an experienced rheumatology consultant were classified as JHS based on the Brighton criteria [24] (Supplementary Table 1) (www.gastrores.org). The remaining patients were classified as non-JHS.

\section{Lactose or fructose intolerance}

Breath tests were carried out to diagnose lactose and fructose malabsorption. In both tests, an increase of $\mathrm{H}_{2}$ concentration $\geq 20 \mathrm{ppm}$ in two consecutive readings above the basal value following ingestion of the test meal was considered lactose intolerance or fructose intolerance [28]. Positive breath tests assist dieticians during the reintroduction phase of the low FODMAP diet intervention, since lactose or fructose intolerance 


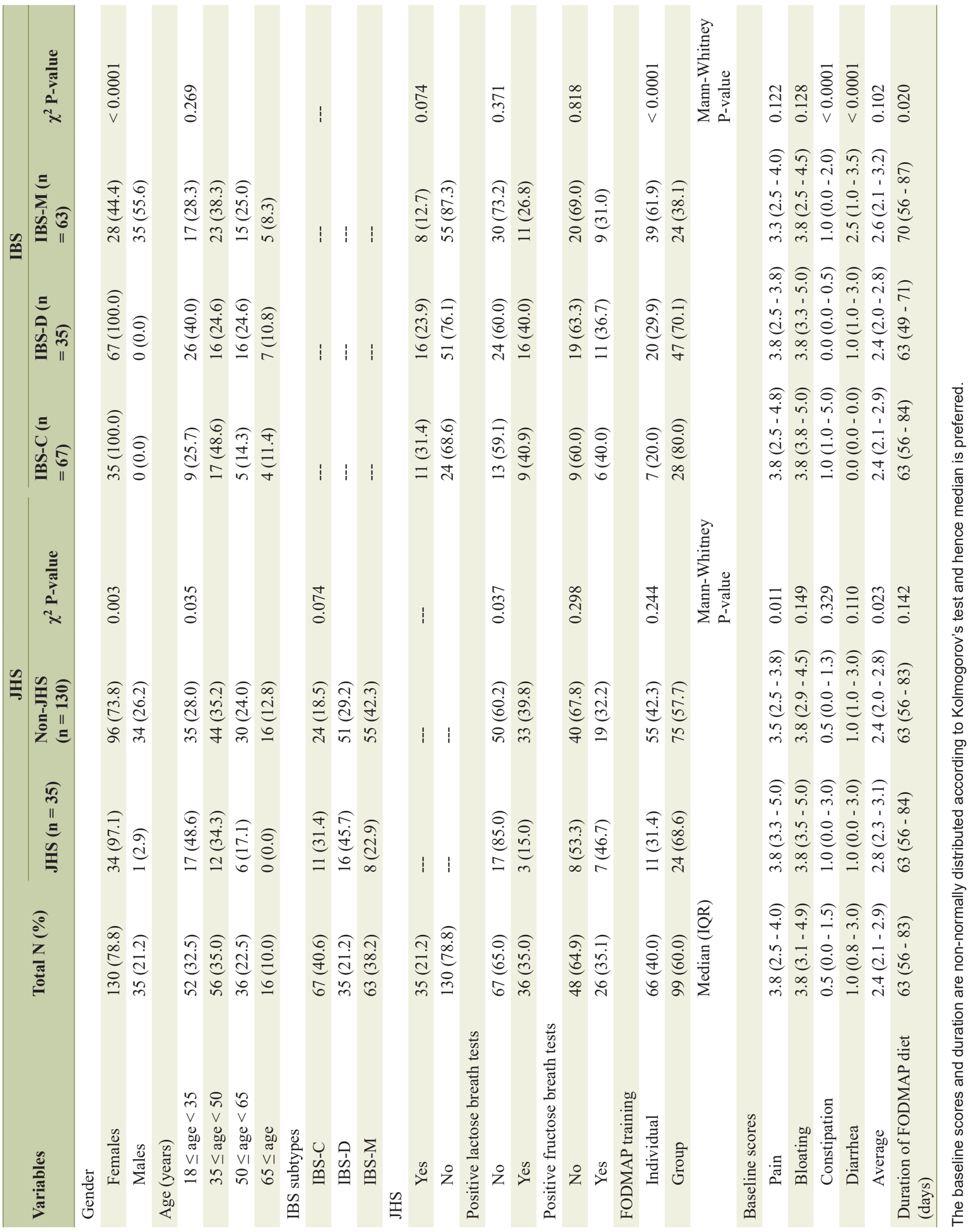


largely precludes their reintroduction into patients' diets [13].

\section{Symptoms score}

Symptom scores for pain, bloating, diarrhea and constipation were measured before and after the dietetic intervention with a visual analogue scale ranging from 0 to 5 . Patients were asked the question: How severe has your "symptom" been over the last 10 days?

\section{Diet}

Patients fulfilling the Rome III criteria for IBS were invited to adhere to the low FODMAP diet. This is recommended by the UK National Institute for Health and Care Excellence (NICE) in the management of patients with IBS [29]. Patients were referred to group or individual guidance on the low FODMAP diet, both led by a trained dietician. Group guidance was carried out with over 10 patients as a group while individual guidance involved a clinic consultation. The process of carrying out the low FODMAP diet involves an informative session with education on restriction of dietary types, helping patients plan menus and encourage patients to develop a strategy plan for the diet ahead.

\section{Statistical Analysis}

Data are presented as mean and standard deviation (SD) or median and interquartile range (IQR) for continuous data and absolute and relative frequency for categorical data, respectively. Univariate analyses were conducted with Chi-square test and Mann-Whitney test for non-normally distributed data. Regression was performed with a mixed effects repeated measures model. For the sake of brevity, we present the coefficients, the significance and the $\mathrm{F}$ tests from of the linear mixed model (type III Wald F tests with Kenward-Roger degrees of freedom approximation). The aim of the model was to investigate temporal trend of symptoms post intervention. Post estimation contrasts were estimated and the effect was presented as the standardized mean difference (SMD) to allow comparability. The strength of association was categorized as following: small, $\mathrm{SMD}=0.2$; medium, $\mathrm{SMD}=0.5$; and large, $\mathrm{SMD}=$ 0.8 [30]. A P-value $\leq 0.05$ was considered significant. For data analysis, IBM SPSS Statistics (Release 22.0.0. 2010, Chicago (IL), USA: SPSS, Inc., an IBM Company) and STATA 14.0 (Stata Corp, College Station, Texas) were used.

\section{Results}

\section{Demographics, JHS and IBS characteristics}

Our sample included 165 patients (130 females) with mean age $44 \pm 14$ years. IBS-D subtype was the most common in our sample (40.6\%) followed by IBS-M (38.2\%) and IBS-C
(21.2\%). Approximately $22 \%$ patients were positive for lactose intolerance and $16 \%$ were positive for fructose intolerance. Thirty-five patients had a positive diagnosis for JHS (21.2\%). Sixty percent of patients received group vs. individual low FODMAP training. The median duration of trialing the low FODMAP was approximately 2 months (63 (56 - 83) days).

Patients with JHS were predominantly female $(\mathrm{P}=0.003)$ and median age was lower than non-JHS patients (JHS: 37 years (27 - 47) vs. non-JHS: 42 years (34 - 56)), Mann-Whitney $\mathrm{P}=0.001)$. The score for pain was higher for JHS compared to non-JHS prior to intervention (JHS: median $3.8(3.3-5.0)$ vs. $3.5(2.5-3.8), \mathrm{P}=0.011)$. No differences were found for bloating, diarrhea, constipation and average scores between both groups at baseline. Also, no differences were found for positive lactose or fructose tests, duration of the low FODMAP diet and whether individual or group training was given. The distribution of symptoms scores according to IBS subtype is shown in Table 1. The highest scores were observed of pain and bloating.

\section{Repeated measures regressions}

The symptoms were analyzed using linear mixed-effects regression, with the xtmixed package in Stata. In contrast to a more traditional approach with data aggregation and repeated-measures analysis of variance analysis, xtmixed allows controlling for the variance associated with random factors without data aggregation [31]. By using random effects for subjects, we controlled for the influence of different mean symptoms associated with the variables JHS and IBS type and their interaction over time. The models are:

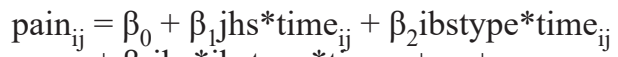

$$
\begin{aligned}
& +\beta_{3}{ }_{j} \text { hs*ibstype*time }{ }_{i j}+u_{j}+\varepsilon_{i j} \\
& \text { bloating }_{\mathrm{ij}}=\beta_{0}+\beta_{1} \mathrm{jhs}^{*} \text { time }_{\mathrm{ij}}+\beta_{2} \text { ibstype }^{*} \text { time }_{\mathrm{ij}} \\
& +\beta_{3}{ }_{j} \text { hs } * \text { ibstype }^{*} \text { time }_{i j}+u_{i j}+\varepsilon_{i j} \\
& \text { diarrhea }_{\mathrm{ij}}=\beta_{0}+\beta_{1} \mathrm{jhs}^{*} \text { time }_{\mathrm{ij}}+\beta_{2} \mathrm{ibstype}^{*} \text { time }_{\mathrm{ij}} \\
& +\beta_{3} \text { jhs }^{*} \text { ibstype }^{*} \text { time }_{\mathrm{ij}}+\mathrm{u}_{\mathrm{j}}+\varepsilon_{\mathrm{ij}} \\
& \text { constipation }_{\mathrm{ij}}=\beta_{0}+\beta_{1} \mathrm{jhs}^{*} \text { time }_{\mathrm{ij}}+\beta_{2}{ }_{\mathrm{ibstype}} * \text { time }_{\mathrm{ij}} \\
& +\beta_{3}{ }_{j} \text { hs*ibstype*time }{ }_{i j}+u_{j}+\varepsilon_{i j} \\
& \text { averscore }_{\mathrm{ij}}=\beta_{0}+\beta_{1} \text { jhs }^{*} \text { time }_{\mathrm{ij}}+\beta_{2} \text { ibstype }^{*} \text { time }_{\mathrm{ij}} \\
& +\beta_{3} \text { jhs*ibstype*time } \mathrm{ij}_{\mathrm{ij}}+\mathrm{u}_{\mathrm{j}}+\varepsilon_{\mathrm{ij}}
\end{aligned}
$$

where $\mathrm{i}=1$ (pre low FODMAP diet), 2 (post low FODMAP diet), $\mathrm{j}=1,2, \ldots, 165$ patients, $\mathrm{u}_{\mathrm{j}}$ is the individual subject effect serving to shift the regression line up or down according to each patient and $\varepsilon_{\mathrm{ij}}$ are the errors of the regression. The reason to choose interaction terms is that JHS and IBS type are significantly associated. The models of each symptom were significant $(\mathrm{P}<0.0001)$. Overall the results of the mixed models are as following.

\section{Pre-post low FODMAP diet}

Symptoms improved in all patients after a low FODMAP diet $(P<0.0001)$ (Tables 2, 3). The largest effect was shown in the significant decrease of average score (SMD $=-2.05$ (95\% CI: 
Table 2. Mixed Model Results for Each Symptom

\begin{tabular}{|c|c|c|c|c|c|c|c|c|c|c|}
\hline & \multicolumn{2}{|c|}{ Pain } & \multicolumn{2}{|c|}{ Bloating } & \multicolumn{2}{|c|}{ Diarrhea } & \multicolumn{2}{|c|}{ Constipation } & \multicolumn{2}{|c|}{ Average score } \\
\hline & beta & $\mathbf{P}$ & beta & $\mathbf{P}$ & beta & $\mathbf{P}$ & beta & $\mathbf{P}$ & beta & $\mathbf{P}$ \\
\hline \multicolumn{11}{|l|}{ JHS \# time } \\
\hline Yes \# pre low FODMAP diet & 0.70 & 0.028 & 0.48 & 0.128 & -0.48 & 0.151 & -0.17 & 0.597 & 0.14 & 0.467 \\
\hline Yes \# post low FODMAP diet & -0.77 & 0.064 & -1.07 & 0.009 & -1.3 & 0.003 & 0.60 & 0.154 & -0.63 & 0.009 \\
\hline IBS-C \# pre low FODMAP diet & 0.06 & 0.064 & 0.27 & 0.327 & -2.03 & $<0.0001$ & 1.96 & $<0.0001$ & 0.06 & 0.686 \\
\hline IBS-C \# post low FODMAP diet & 0.05 & 0.049 & 0.33 & 0.221 & -0.99 & $<0.0001$ & -0.02 & 0.943 & -0.16 & 0.319 \\
\hline IBS-M \# pre low FODMAP diet & -0.07 & -0.073 & -0.04 & 0.835 & 0.18 & 0.416 & 0.98 & $<0.0001$ & 0.26 & 0.037 \\
\hline IBS-M \# post low FODMAP diet & 0 & omitted & 0 & omitted & 0 & omitted & 0 & omitted & 0 & omitted \\
\hline \multicolumn{11}{|l|}{ JHS \#IBS Type \# time } \\
\hline Yes \# IBS-M \# pre low FODMAP diet & -0.97 & 0.067 & -0.75 & 0.152 & 0.91 & 0.097 & 1.58 & 0.003 & 0.19 & 0.535 \\
\hline Yes \# IBS-M \# post low FODMAP diet & -0.92 & 0.085 & -0.65 & 0.213 & -0.34 & 0.540 & 1.07 & 0.044 & -0.21 & 0.498 \\
\hline Constant & 3.31 & $<0.0001$ & 3.63 & $<0.0001$ & 1.81 & $<0.0001$ & 0.20 & 0.200 & 2.32 & $<0.0001$ \\
\hline F test & $<0.00$ & & $<0.00$ & & $<0.00$ & & $<0.00$ & & $<0.0$ & \\
\hline
\end{tabular}

$-2.42,-1.67))$ followed by a significant decrease in bloating $(\mathrm{SMD}=-1.50,(95 \% \mathrm{CI}:-1.85,-1.16))$.

\section{Impact on IBS symptoms with regard to the presence of JHS}

Symptoms improved post low FODMAP diet in both JHS and non-JHS patients $(\mathrm{P}<0.001)$ (Table 3$)$. However, effects were more prominent in the JHS group with larger SMDs for each symptom in the JHS group compared to the non-JHS group (Figs. 1, 2). For example, pain improved with a larger effect in JHS patients compared to non-JHS patients (SMD -1.89 vs. -1.38 , respectively) (Table 3 ).

\section{Impact on IBS symptoms with reference to IBS type}

Symptoms improved significantly with post low FODMAP diet in all IBS subgroups (Tables 2, 3). The low FODMAP diet significantly improved pain, bloating, diarrhea and the average score with the largest effect in the IBS-D group (SMDs $=-2.01,(95 \%$ CI: $-2.84,-1.19),-1.98$ (95\% CI: $-2.80,-1.17)$, -2.51 (95\% CI: $-3.41,-1.61)$, and -2.70 (95\% CI: -3.63, -1.77), respectively), while constipation improved with the largest effect in the IBS-C group (SMD $=-1.35,(95 \% \mathrm{CI}:-1.88,-0.81)$ ).

Impact on IBS symptoms when considering interaction of JHS and IBS type

When broken down by JHS and IBS type, most symptoms im- proved significantly post low FODMAP diet (Table 3). A low FODMAP diet significantly improved pain with the largest effect in the JHS/IBS-C group (SMD =-2.62, (95\% CI: -4.31 , -0.93)); significantly improved bloating with the largest effect in the JHS/IBS-M group (SMD = -2.41, (95\% CI: $-4.36,-0.47)$ ); significantly improved diarrhea with the largest effect in the JHS/IBS-D group (SMD = -4.00, (95\% CI: -6.66, -1.35)); significantly improved constipation with the largest effect in the nonJHS/IBS-C group (SMD = -2.23, (95\% CI: -3.27, -1.19)); and significantly improved the average score with the largest effect in the JHS/IBS-M group (SMD = -4.33, (95\% CI: -7.15, -1.52)). Overall, JHS seems to affect response to symptoms mainly with regards to pain, bloating, diarrhea and the average score.

Although there was no gender difference in response for JHS individuals with IBS, female patients with JHS exhibited larger effects for all symptoms compared to non-JHS patients (Supplementary Tables 2, 3) (www.gastrores.org).

\section{Discussion}

To our knowledge, this is the first study to investigate response to the low FODMAP diet in patients with IBS and co-existent congenital connective tissue disorder, JHS which has been associated with frequent and more severe functional gut disorders. Our findings show that JHS patients have significant improvement in their pain, bloating, diarrhea and constipation after the low FODMAP diet. The overall symptom score improvement for both JHS and non-JHS patients were statistically significant which stated that the low FODMAP diet is effective in both JHS and non-JHS group. However, IBS symptoms demonstrated a 


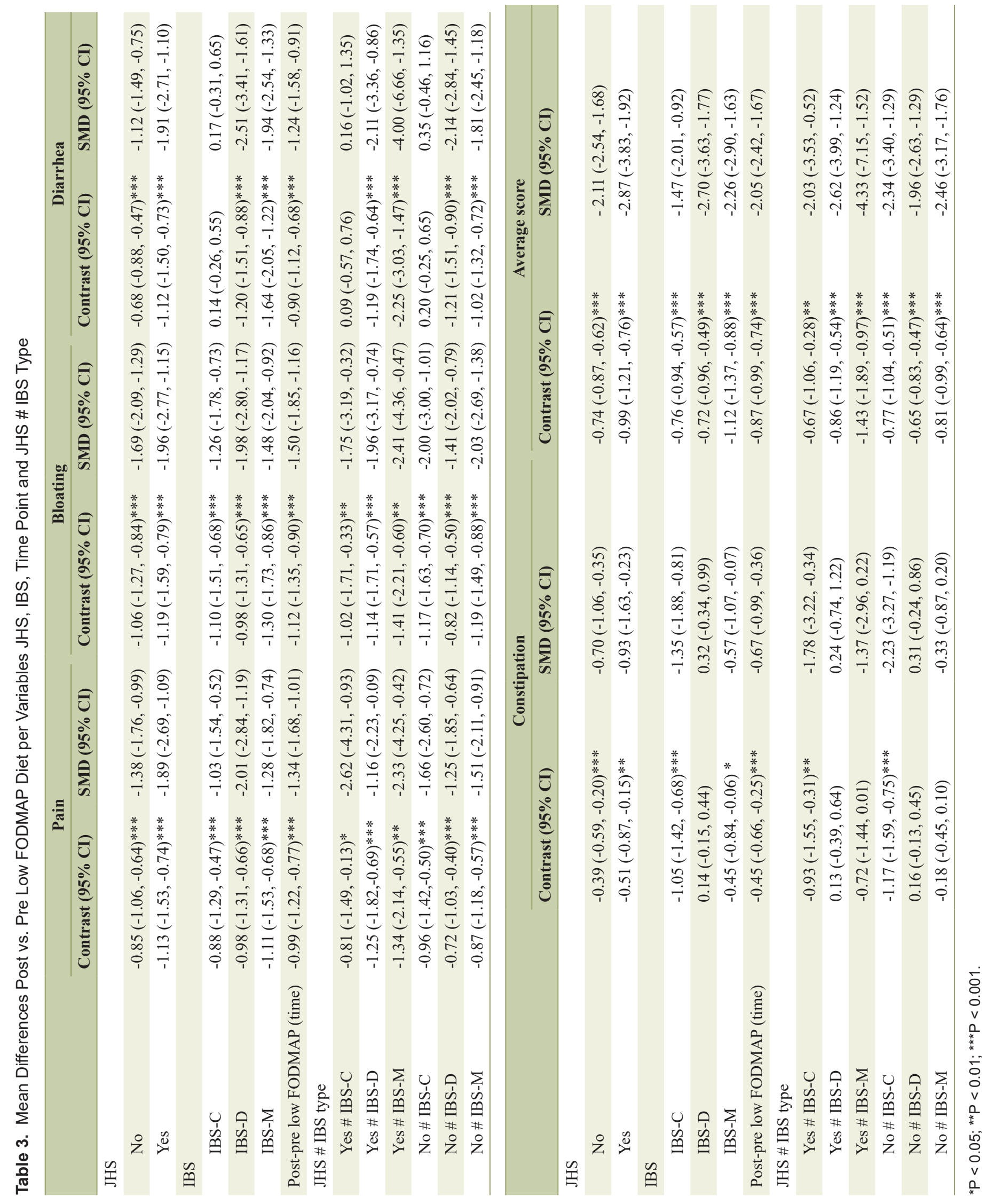



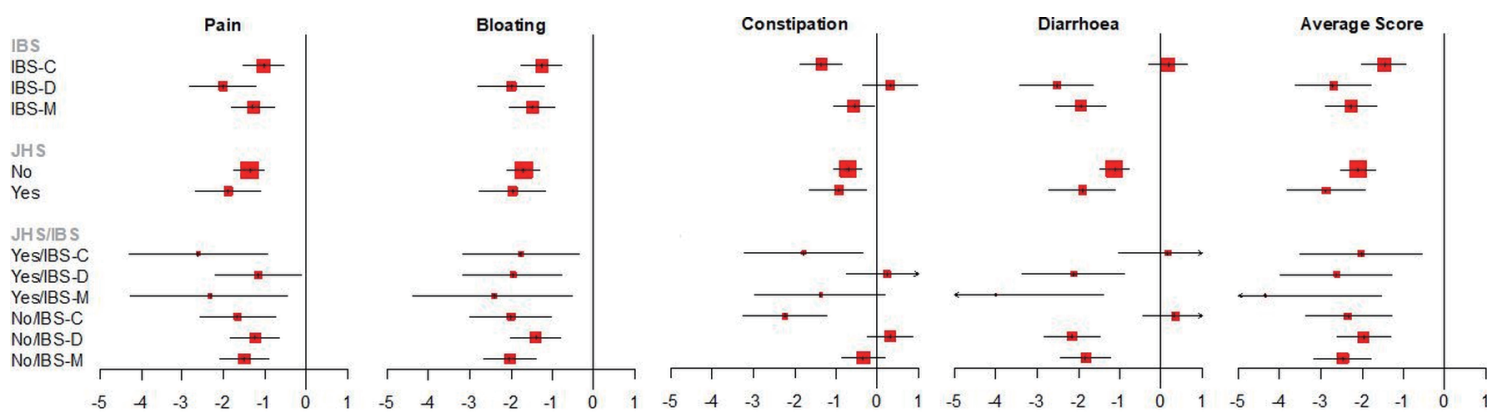

Figure 1. Effect sizes (SMD), according to JHS and IBS and combination.

bigger improvement in JHS patients. Researchers have found bloating, abdominal pain and diarrhea are the symptoms that improve significantly on a low FODMAP diet, whereas constipation was often reported to non-significantly improve or not improve $[32,33]$. A low FODMAP diet likely reduces osmotic fluid transit into the gut lumen and consequently increases the chances of having constipation. Interestingly, our study demonstrated significant treatment efficacy of a low FODMAP diet in the constipation symptom irrespective of JHS status in IBS-C and a non-significant decrease in IBS-M.

Pain mechanisms in JHS patients remain poorly understood. In the present study, baseline pain scores were significantly higher in patients with JHS compared to non-JHS patients (median 3.8 vs. 3.5, respectively) with JHS patients responding with a larger effect compared to non-JHS patients (SMD -1.89 vs. -1.39, respectively). Pathophysiological models of JHS suggest nociceptive and neuropathic components, as well as pain sensitization. Pain in IBS is precipitated by negative emotions and expectations playing an important role in pain evaluation. Pain processing in IBS is mediated by functional and structural brain changes with peripheral neuroendocrine and immune pathways are also relevant in IBS, which is similar to pain mechanisms in JHS [34]. Prevalence of psychological comorbidity, particularly anxiety, has been described in JHS and might be a contributing factor to increase body awareness and pain related unhelpful behaviors [35]. The present study suggests that overall pain could be a result of additive processes in the co-existence of IBS and JHS and hence the low FODMAP may reduce the burden of pain in these patients.

Our sample included approximately $20 \%$ of JHS patients. The majority of them were female and in the second and third decade of age. JHS patients frequently report abdominal pain and severe constipation [36], and this was replicated in the present study. It is possible that increased prevalence of chronic pain syndromes might be related to hormonal status and increased joint laxity in younger ages, increased prevalence of anxiety and depression in the female population, and altered pain processing with heighted body awareness [37]. Constipation has been found to be particularly prevalent in both the pediatric and adult population with co-existent JHS disorder [38]. Although the mechanism is unknown, it is possible that abnormal connective tissue predisposes to altered colonic transit or it could be that the frequent described autonomic dysfunction contributes to colonic dysfunction. Hence, greater ef- fectiveness of the low FODMAP diet in this group of patients might be secondary to a greater degree of abdominal discomfort relieved in patients with visceral hypersensitivity.

Some studies suggest that the low FODMAP diet is likely to reduce diarrhea but might not improve constipation in patients with IBS [39]. A recent audit surveyed 17 patients and found that $82 \%$ reported positive relief of gastrointestinal symptoms [40]. Shepherd et al evaluated in a randomized controlled trial the clinical response in 25 patients with IBS with fructose malabsorption demonstrated on a hydrogen breath test [41]. The study proved that low FODMAPs diet could reduce symptoms in IBS patients, particularly in patients with fructose malabsorption. In a recent control and crossover study, patients were exposed to a low FODMAPs diet for 21 days or the typical Australian diet with at least one food high in FODMAP [11]. Gastrointestinal symptoms were significantly different between the two diets and the greatest symptom control was achieved and maintained after 7 days of the low FODMAP diet.

The low FODMAP diet in this study was adhered to by patients for over 8 weeks on average, which is in line with the suggested $6-8$ weeks $[10,13]$. Most patients received group training compared to the preferred individual training. Although the distribution of training type was equal among JHS and non-JHS patients, IBS-M patients were more likely to receive individual training than group training and vice versa for IBS-C and IBS-D. A limitation exists with respect to the gender distribution in the JHS group, which was skewed towards a female population predominantly. When the effect of JHS is broken down by gender, then all symptoms were not affected by a low FODMAP for male patients with JHS. Female patients with JHS exhibited larger effects for all symptoms compared to non-JHS patients (Supplementary Tables 2, 3) (www.gastrores.org). Another main limitation involves selection bias, since the included JHS patients were those seeking specialist care in tertiary rheumatology clinics; it is unclear if this population represents patients with JHS in the community. However, it is important to recognize that all IBS patients were referred from a neurogastroenterology clinic and likely represent a cohort of patients with more severe symptoms than the general population. Methodological limitations included the retrospective design of the study, the use of a non-validated symptom measurement tool and the fact that statistical analyses included regression to the mean which can bias any investigation where the response to treatment is classified relative 


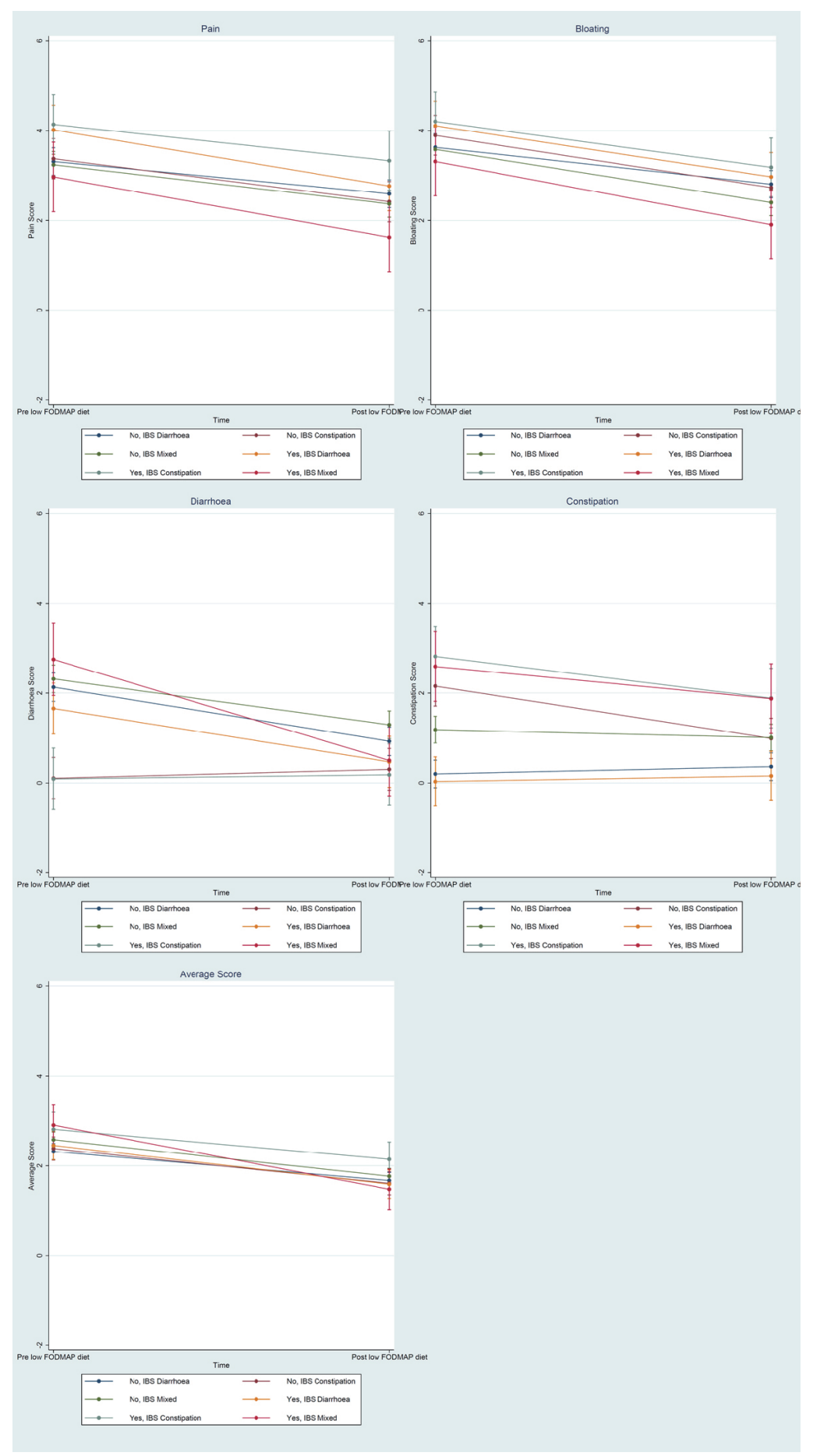

Figure 2. Margins plot for pain, bloating, diarrhea, constipation and average score.

to initial values for a given variable without the use of an appropriate control group [42].

\section{Conclusions}

In conclusion, with the increasing recognition of JHS, more patients from this cohort will be referred to the gastroenterology clinic. Our study suggests that a low FODMAP diet has greater improvement in JHS patients than non-JHS patients concerning IBS symptoms (pain, diarrhea, bloating and constipation). Treatment for JHS patients is hence necessary to reduce the gastrointestinal symptoms and a low FODMAP diet should be recommended. Future studies need to focus on the 
association of gastrointestinal symptoms in JHS patients in order to approach more suitable treatment on IBS patients with JHS.

\section{Declaration}

Any research materials related to the present manuscript (for example datasets or models) can be obtained by contacting the corresponding author.

\section{Conflict of Interest}

The authors declare that there is no conflict of interest.

\section{Funding Disclosure}

The preparation of this review was not supported by any external funding.

\section{References}

1. Kim HP, Crockett SD, Shaheen NJ. The Burden of Gastrointestinal and Liver Disease Around the World. In: Talley NJ, Locke GR, III, Moayyedi P, West J, Ford AC, Saito YA, editors. GI Epidemiology: Diseases and Clinical Methodology. 2nd ed. Chichester, West Sussex: John Wiley \& Sons; 2014. p. 1-13.

2. Van Oudenhove L, Levy RL, Crowell MD, Drossman DA, Halpert AD, Keefer L, Lackner JM, et al. Biopsychosocial aspects of functional gastrointestinal disorders. Gastroenterology. 2016;150(6):1355-1367, e2.

3. Mearin F, Lacy BE, Chang L, Chey WD, Lembo AJ, Simren M, et al. Bowel Disorders. Gastroenterology. 2016;150(6):1393-407.e5.

4. Yao X, Yang YS, Cui LH, Zhao KB, Zhang ZH, Peng LH, Guo X, et al. Subtypes of irritable bowel syndrome on Rome III criteria: a multicenter study. J Gastroenterol Hepatol. 2012;27(4):760-765.

5. Engsbro AL, Simren M, Bytzer P. Short-term stability of subtypes in the irritable bowel syndrome: prospective evaluation using the Rome III classification. Aliment Pharmacol Ther. 2012;35(3):350-359.

6. Fragkos KC. Spotlight on eluxadoline for the treatment of patients with irritable bowel syndrome with diarrhea. Clin Exp Gastroenterol. 2017;10:229-240.

7. Barbara G, Feinle-Bisset C, Ghoshal UC, Quigley EM, Santos J, Vanner S, Vergnolle N, et al. The Intestinal Microenvironment and Functional Gastrointestinal Disorders. Gastroenterology. 2016;150(6):1305-1318, e8.

8. Chey WD, Whelan K. Dietary guidelines for irritable bowel syndrome are important for gastroenterologists, dietitians and people with irritable bowel syndrome. J Hum Nutr Diet. 2016;29(5):547-548.

9. Masuy I, Van Oudenhove L, Tack J, Biesiekierski JR.
Effect of intragastric FODMAP infusion on upper gastrointestinal motility, gastrointestinal, and psychological symptoms in irritable bowel syndrome vs healthy controls. Neurogastroenterol Motil. 2018;30(1):e13167.

10. Rao SS, Yu S, Fedewa A. Systematic review: dietary fibre and FODMAP-restricted diet in the management of constipation and irritable bowel syndrome. Aliment Pharmacol Ther. 2015;41(12):1256-1270.

11. Halmos EP, Power VA, Shepherd SJ, Gibson PR, Muir JG. A diet low in FODMAPs reduces symptoms of irritable bowel syndrome. Gastroenterology. 2014;146(1):6775 e 65.

12. Gibson PR. The evidence base for efficacy of the low FODMAP diet in irritable bowel syndrome: is it ready for prime time as a first-line therapy? J Gastroenterol Hepatol. 2017;32(Suppl 1):32-35.

13. Whelan K, Martin LD, Staudacher HM, Lomer MCE. The low FODMAP diet in the management of irritable bowel syndrome: an evidence-based review of FODMAP restriction, reintroduction and personalisation in clinical practice. J Hum Nutr Diet. 2018;31(2):239-255.

14. O'Keeffe M, Jansen C, Martin L, Williams M, Seamark L, Staudacher HM, Irving PM, et al. Long-term impact of the low-FODMAP diet on gastrointestinal symptoms, dietary intake, patient acceptability, and healthcare utilization in irritable bowel syndrome. Neurogastroenterol Motil. 2018;30(1):e13154.

15. Altobelli E, Del Negro V, Angeletti PM, Latella G. LowFODMAP Diet Improves Irritable Bowel Syndrome Symptoms: A Meta-Analysis. Nutrients. 2017;9(9):940.

16. Fikree A. Functional Gastrointestinal Disorders and the Joint Hypermobility Syndrome. Queen Mary University of London; 2013.

17. Botrus G, Baker O, Borrego E, Ngamdu KS, Teleb M, Gonzales Martinez JL, Maldonado G, 3rd, et al. Spectrum of Gastrointestinal Manifestations in Joint Hypermobility Syndromes. Am J Med Sci. 2018;355(6):573-580.

18. Castori M, Morlino S, Pascolini G, Blundo C, Grammatico P. Gastrointestinal and nutritional issues in joint hypermobility syndrome/Ehlers-Danlos syndrome, hypermobility type. Am J Med Genet C Semin Med Genet. 2015;169C(1):54-75.

19. Kumar B, Lenert P. Joint hypermobility syndrome: recognizing a commonly overlooked cause of chronic pain. Am J Med. 2017;130(6):640-647.

20. Beckers AB, Keszthelyi D, Fikree A, Vork L, Masclee A, Farmer AD, Aziz Q. Gastrointestinal disorders in joint hypermobility syndrome/Ehlers-Danlos syndrome hypermobility type: A review for the gastroenterologist. Neurogastroenterol Motil. 2017;29(8):e13013.

21. Forghani I. Updates in clinical and genetics aspects of hypermobile Ehlers Danlos syndrome. Balkan Med J. 2019;36(1):12-16.

22. Colombi M, Dordoni C, Chiarelli N, Ritelli M. Differential diagnosis and diagnostic flow chart of joint hypermobility syndrome/ehlers-danlos syndrome hypermobility type compared to other heritable connective tissue disorders. Am J Med Genet C Semin Med Genet. 2015;169C(1):622. 
23. Malfait F, Francomano C, Byers P, Belmont J, Berglund B, Black J, Bloom L, et al. The 2017 international classification of the Ehlers-Danlos syndromes. Am J Med Genet C Semin Med Genet. 2017;175(1):8-26.

24. Fikree A, Aziz Q, Grahame R. Joint hypermobility syndrome. Rheum Dis Clin North Am. 2013;39(2):419-430.

25. Zarate N, Farmer AD, Grahame R, Mohammed SD, Knowles CH, Scott SM, Aziz Q. Unexplained gastrointestinal symptoms and joint hypermobility: is connective tissue the missing link? Neurogastroenterol Motil. 2010;22(3):252-e278.

26. Fikree A, Aktar R, Grahame R, Hakim AJ, Morris JK, Knowles CH, Aziz Q. Functional gastrointestinal disorders are associated with the joint hypermobility syndrome in secondary care: a case-control study. Neurogastroenterol Motil. 2015;27(4):569-579.

27. von Elm E, Altman DG, Egger M, Pocock SJ, Gotzsche PC, Vandenbroucke JP, Initiative S. The Strengthening the Reporting of Observational Studies in Epidemiology (STROBE) statement: guidelines for reporting observational studies. J Clin Epidemiol. 2008;61(4):344-349.

28. Rana SV, Malik A. Breath tests and irritable bowel syndrome. World J Gastroenterol. 2014;20(24):7587-7601.

29. Irritable bowel syndrome in adults. Diagnosis and management of irritable bowel syndrome in primary care. National Institute for Health and Clinical Excellence: Guidance. London. 2008.

30. Fragkos KC, Zarate-Lopez N, Frangos CC. What about clonidine for diarrhoea? A systematic review and metaanalysis of its effect in humans. Therap Adv Gastroenterol. 2016;9(3):282-301.

31. Baayen RH, Davidson DJ, Bates DM. Mixed-effects modeling with crossed random effects for subjects and items. J Mem Lang. 2008;59(4):390-412.

32. Staudacher HM, Whelan K, Irving PM, Lomer MC. Comparison of symptom response following advice for a diet low in fermentable carbohydrates (FODMAPs) versus standard dietary advice in patients with irritable bowel syndrome. J Hum Nutr Diet. 2011;24(5):487-495.

33. Bohn L, Storsrud S, Liljebo T, Collin L, Lindfors P, Tornblom H, Simren M. Diet low in FODMAPs reduces symptoms of irritable bowel syndrome as well as tradi- tional dietary advice: a randomized controlled trial. Gastroenterology. 2015;149(6):1399-1407 e1392.

34. Elsenbruch S. Abdominal pain in Irritable Bowel Syndrome: a review of putative psychological, neural and neuro-immune mechanisms. Brain Behav Immun. 2011;25(3):386-394.

35. Baeza-Velasco C, Bourdon C, Montalescot L, de Cazotte C, Pailhez G, Bulbena A, Hamonet C. Low- and highanxious hypermobile Ehlers-Danlos syndrome patients: comparison of psychosocial and health variables. Rheumatol Int. 2018;38(5):871-878.

36. Mohammed SD, Lunniss PJ, Zarate N, Farmer AD, Grahame R, Aziz Q, Scott SM. Joint hypermobility and rectal evacuatory dysfunction: an etiological link in abnormal connective tissue? Neurogastroenterol Motil. 2010;22(10):1085-e1283.

37. Scheper MC, de Vries JE, Verbunt J, Engelbert RH. Chronic pain in hypermobility syndrome and EhlersDanlos syndrome (hypermobility type): it is a challenge. J Pain Res. 2015;8:591-601.

38. Reilly DJ, Chase JW, Hutson JM, Clarke MC, Gibb S, Stillman B, Southwell BR. Connective tissue disorder a new subgroup of boys with slow transit constipation? J Pediatr Surg. 2008;43(6):1111-1114.

39. Gearry RB, Irving PM, Barrett JS, Nathan DM, Shepherd SJ, Gibson PR. Reduction of dietary poorly absorbed short-chain carbohydrates (FODMAPs) improves abdominal symptoms in patients with inflammatory bowel disease-a pilot study. J Crohns Colitis. 2009;3(1):8-14.

40. Kinrade SK, Twamley RM, Fell L, Heald L, Healy A. PTH-066 an audit to assess feasibility and efficacy of group education for Irritable Bowel Syndrome (IBS) Patients in the Delivery of Low FODMAP (Fermentable Oligosaccharides, Disaccharides, Monosaccharides And Polyols) Dietary Advice. Gut. 2014;63(Suppl 1):A238.1.

41. Shepherd SJ, Parker FC, Muir JG, Gibson PR. Dietary triggers of abdominal symptoms in patients with irritable bowel syndrome: randomized placebo-controlled evidence. Clin Gastroenterol Hepatol. 2008;6(7):765-771.

42. Barnett AG, van der Pols JC, Dobson AJ. Regression to the mean: what it is and how to deal with it. Int J Epidemiol. 2005;34(1):215-220. 